\title{
Solvating atomic level fine-grained proteins in supra-molecular level coarse-grained water for molecular dynamics simulations
}

\author{
Journal Article \\ Author(s): \\ Riniker, Sereina; Eichenberger, Andreas P.; van Gunsteren, Wilfred F. \\ Publication date: \\ 2012 \\ Permanent link: \\ https://doi.org/10.3929/ethz-b-000054564
}

Rights / license:

In Copyright - Non-Commercial Use Permitted

Originally published in:

European Biophysics Journal 41(8), https://doi.org/10.1007/s00249-012-0837-1 


\title{
Solvating atomic level fine-grained proteins in supra-molecular level coarse-grained water for molecular dynamics simulations
}

\author{
Sereina Riniker • Andreas P. Eichenberger • \\ Wilfred F. van Gunsteren
}

Received: 9 May 2012/Revised: 18 June 2012/ Accepted: 25 June 2012/Published online: 14 July 2012

(C) European Biophysical Societies' Association 2012

\begin{abstract}
Simulation of the dynamics of a protein in aqueous solution using an atomic model for both the protein and the many water molecules is still computationally extremely demanding considering the time scale of protein motions. The use of supra-atomic or supra-molecular coarse-grained (CG) models may enhance the computational efficiency, but inevitably at the cost of reduced accuracy. Coarse-graining solvent degrees of freedom is likely to yield a favourable balance between reduced accuracy and enhanced computational speed. Here, the use of a supra-molecular coarse-grained water model that largely preserves the thermodynamic and dielectric properties of atomic level fine-grained (FG) water in molecular dynamics simulations of an atomic model for four proteins is investigated. The results of using an FG, a CG, an implicit, or a vacuum solvent environment of the four proteins are compared, and for hen egg-white lysozyme a comparison to NMR data is made. The mixed-grained simulations do not show large differences compared to the FG atomic level simulations, apart from an increased tendency to form hydrogen bonds between long side chains, which is due to the reduced ability of the supra-molecular CG beads that represent five FG water molecules to make solvent-protein hydrogen bonds. But, the mixed-grained simulations are at least an order of magnitude faster than the atomic level ones.
\end{abstract}

Keywords Coarse-graining - Molecular dynamics . Protein - Water · Multigraining

S. Riniker · A. P. Eichenberger · W. F. van Gunsteren ( $\square)$ Laboratory of Physical Chemistry, ETH Zurich, 8093 Zurich, Switzerland

e-mail:wfvgn@igc.phys.chem.ethz.ch

\section{Introduction}

To describe the dynamic processes of biomolecules such as proteins and peptides, atomic level molecular dynamics (MD) simulations have been proven to be a useful method. Although the increase of computer power has given access to larger and larger system sizes and time scales, MD simulations are generally still limited to the multi-nanometer and multi-nanosecond range nowadays. As most biological processes occur on larger time scales, the development of models at a more coarse-grained (CG) level of resolution to reduce the computational effort is of considerable interest. In the process of coarse graining, a chosen number of atoms is subsumed into a single CG bead, thus reducing the number of particle-particle interactions. In addition, the generally smoother interaction function between CG beads facilitates the sampling of configurations. However, the choice of the degrees of freedom to be eliminated in the process of coarse-graining is not trivial and depends largely on the questions that should be answered using the CG model, as coarse graining involves per se a loss of information. Generally, it is advantageous to select degrees of freedom that account for a large part of the computational cost. Coarse graining of these provides considerable speed-up of the simulation, while these may be of less interest for the property or biomolecular process to be investigated. To this category we count the solvent degrees of freedom and, in the case of membrane proteins, also intra-molecular degrees of freedom of lipids, whereas proteins usually present only a small part of the system and a detailed, i.e. atomistic, description is often essential for a correct representation of their structural and dynamic properties. Thus, combining an atomistic, fine-grained (FG) protein with a CG solvent may be a promising approach to simultaneously reduce the 
computational cost of a simulation and retain the details for the region of interest, i.e. the protein. A variety of multigraining schemes has been proposed recently [see Ref. Riniker and van Gunsteren (2012) for an overview]. Here, we focus on so-called hybrid systems of FG and CG particles where the FG and CG levels of modelling are combined analogous to hybrid quantum/classical (QM/MM) models (Neri et al. 2005; Shi et al. 2006; Michel et al. 2008; Masella et al. 2008, 2011; Orsi et al. 2009; Rzepiela et al. 2011). Thereby, molecules of different resolution, i.e. FG or CG, coexist in the system and the resolution of a molecule is fixed. The proposed hybrid approaches differ in the central question of how to define the interaction between the FG and $\mathrm{CG}$ particles.

Shi et al. (2006) chose to completely reparametrise all FG-CG interactions using the force-matching methodology. They applied their model to study the polypetide gramicidin A forming an $\alpha$-helix in a CG DMPC membrane solvated in CG water. Thereby, a single water molecule is represented by a CG water bead and in the lipids groups of 3-6 atoms are subsumed into $\mathrm{CG}$ beads. The backbone atom-positional RMSD of the polypetide from the NMR solution model structure in the $10 \mathrm{~ns}$ mixed-grained simulation agrees with the RMSD of the purely atomistic simulation.

Michel et al. (2008) employ a standard 12-6 LennardJones potential energy function for the CG beads and thus the FG-CG Lennard-Jones interaction parameters can be derived using standard combination rules. Two additional parameters were introduced to calibrate the FG-CG Lennard-Jones and electrostatic interactions to reproduce experimental partition coefficients of amino-acid sidechain analogues. As CG solvent the soft sticky dipole model (Liu and Ichiye 1996) was used, which represents a single water molecule. They also applied their model to study the transmembrane permeability of a series of small organic molecules through a CG DMPC membrane.

Another approach has been proposed by Masella et al. (2008, 2011) where the polarisable pseudo-particle water model (Basdevant et al. 2006) is mixed with a polarisable atomistic solute model. They found that the standard Lennard-Jones potential energy term was not hard enough to prevent a polarisation catastrophe and had to be altered accordingly. Their solvent model represents a single water molecule, where the solvent dipoles only interact with the solute dipoles and not with each other. For the mixed simulations, a model-dependent renormalisation factor had to be introduced for the dipoles of the solute. The model has been applied to study the structural stability of bovine pancreatic trypsin inhibitor (BPTI) (Masella et al. 2008, 2011), as well as G-protein and a ubiquitin-like domain (Masella et al. 2011) by analysing the atom-positional RMSD of the backbone from the X-ray structure and the potential energy terms.
A fourth method avoids the definition of FG-CG interactions altogether by introducing virtual $\mathrm{CG}$ sites for groups of FG atoms, where the CG solvent only interacts with these virtual CG sites (Rzepiela et al. 2011). The forces of the $\mathrm{CG}$ virtual sites are then mapped back on the underlying FG atoms. The model has been tested on FG butane molecules in CG butane and on FG dialanine peptides in $\mathrm{CG}$ water or $\mathrm{CG}$ butane. Thereby, four water molecules are represented by a Lennard-Jones bead without electrostatic interactions.

Most of the hybrid models discussed above employ a CG solvent model that represents only a single water molecule, thus limiting the achievable speed-up. Here, we use a recently introduced CG water model (Riniker and van Gunsteren 2011) where five water molecules are subsumed into a CG bead with explicit electrostatic interactions, thus reproducing the dielectric screening properties of water. The application of this model in mixed-grained simulations has been investigated for FG/CG mixtures of liquid water (Riniker and van Gunsteren 2012). Only a minor parametrisation effort was required to reproduce the solvation free energy of water and the solvation free energies of small alkanes in water. Here, we test the generality of this mixedgrained model on four proteins, i.e. hen egg-white lysozyme (HEWL), major cold shock protein (CspA), G-protein (GP), and chorismate mutase (CM), solvated in $\mathrm{CG}$ water at room temperature and pressure. The four proteins represent different secondary structure motifs, i.e. all $\alpha$-helical, all $\beta$-strand, and combinations thereof. The structural and dynamic properties are analysed and compared to those obtained from purely atomic level simulations, from simulations using an implicit solvent or in vacuo. In addition, the configurational ensemble of hen egg-white lysozyme is used for a comparison of simulated averages to experimental data for a number of observable quantities.

\section{Methods}

Fine-grained and coarse-grained models

The atomistic GROMOS force field (Schmid et al. 2011) 54A7 was used for the proteins, and the simple-pointcharge model (Berendsen et al. 1981) (SPC) as atomistic fine-grained solvent. In the coarse-grained solvent model, five SPC water molecules are subsumed into a spherical CG bead, which consists of two interaction sites, named "center water" (CW) and "dipole particle" (DP), representing a polarisable dipole. The two sites are connected by an unconstrained bond with an attractive quartic potential energy function, making the model polarisable. This CG model is described in detail in Ref. Riniker and van Gunsteren (2011). 
Fig. 1 From left to right, crystal structures of hen eggwhite lysozyme [HEWL, PDB entry 1AKI (Artymiuk et al. 1982)], major cold shock protein [CspA, PDB entry 1MJC (Schindelin et al. 1994)], protein $\mathrm{G}$ [GP, PDB entry $1 \mathrm{PGB}$ (Gallagher et al. 1994)], and chorismate mutase [CM, PDB entry 2FP2 (Okvist et al. 2006)]. $\alpha$-helices are shown in red, $3_{10}$-helices in black, and $\beta$-strands in blue

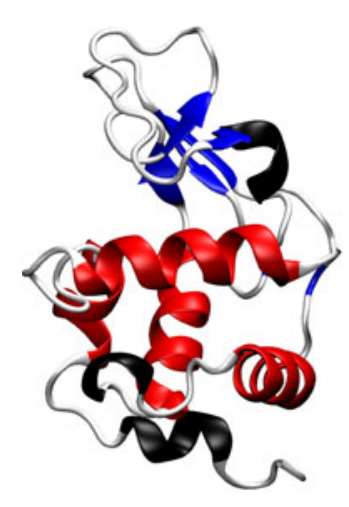

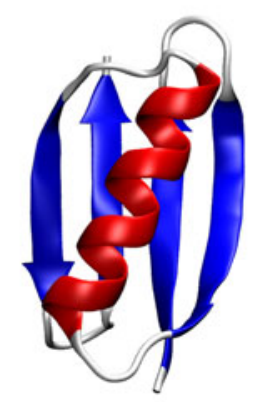

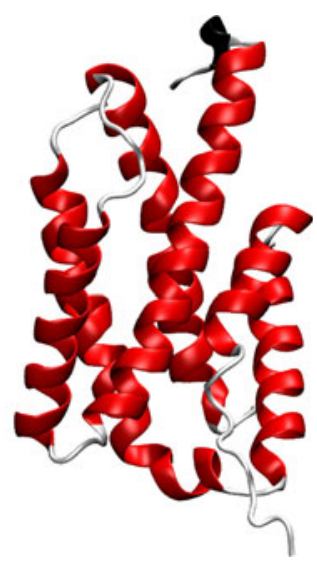

The Lennard-Jones parameters for the FG-CG interactions are determined using the standard GROMOS combination rules (van Gunsteren et al. 1996). The DP site of the CG bead, which interacts purely electrostatically with other $\mathrm{CG}$ beads, has an additional repulsive $C_{12}$ term for the interaction with $\mathrm{FG}$ atoms to prevent a polarisation catastrophe. The dielectric permittivity within the cutoff sphere is $\varepsilon_{\mathrm{cs}}^{\mathrm{FG}}=1$ for $\mathrm{FG}-\mathrm{FG}$ electrostatic interactions, $\varepsilon_{\mathrm{cs}}^{\mathrm{mix}}=2.3$ for FG-CG interactions, and $\varepsilon_{\mathrm{cs}}^{\mathrm{CG}}=2.5$ for CG$\mathrm{CG}$ interactions. The parametrisation of the FG-CG interactions is described in more detail in Ref. Riniker and van Gunsteren (2012).

\section{Proteins}

The four proteins were chosen to cover the diverse secondary and tertiary structure elements commonly found, i.e. purely $\alpha$-helical, purely $\beta$-strand, and combinations thereof. The proteins were hen egg-white lysozyme [HEWL, RCSB protein databank (PDB) entry 1AKI (Artymiuk et al. 1982)], major cold shock protein [CspA, PDB entry 1MJC (Schindelin et al. 1994)], protein G [GP, PDB entry 1PGB (Gallagher et al. 1994)], and chorismate mutase [CM, PDB entry 2FP2 (Okvist et al. 2006)] (Fig. 1). The protonation states were chosen to match a $\mathrm{pH}$ of 7 and hydrogen atoms were added to the X-ray structures according to standard geometric criteria. The histidine side chains were protonated at $\mathrm{N}_{\delta}$ or $\mathrm{N}_{\varepsilon}$ depending on their hydrogen-bonding environment. In CM, the first three residues were not considered.

\section{Simulation details}

All simulations were performed for 20 ns under NPT conditions using the GROMOS package of programs (Schmid et al. 2012; Kunz et al. 2012). ${ }^{1}$ The bond lengths of the

\footnotetext{
1 The GROMOS package of programs can be obtained from http://www.gromos.net.
}

protein were constrained to the ideal values applying the SHAKE algorithm (Ryckaert et al. 1977). The temperature was maintained close to its reference value $T=298 \mathrm{~K}$ by weak coupling to a temperature bath with a relaxation time of 0.1 ps (Berendsen et al. 1984). The pressure was maintained close to its reference value $P=1.013 \mathrm{bar}$ ( $1 \mathrm{~atm}$ ) by weak coupling to a pressure bath with a relaxation time of $0.5 \mathrm{ps}$ and using the isothermal compressibility $\kappa_{T}=$ $4.575 \times 10^{-4}\left(\mathrm{~kJ} \mathrm{~mol}^{-1} \mathrm{~nm}^{-3}\right)^{-1}$. Newton's equations of motion were integrated using the leap-frog scheme (Hockney 1970) with a time step of 2 fs. A reaction field force (Tironi et al. 1995) was applied using the experimental relative dielectric permittivity $\varepsilon_{\mathrm{rf}}=78.5$ (Lide 2007) in the case of an explicit solvent model, or $\varepsilon_{\mathrm{rf}}=1$ for the simulations using an implicit solvent SASA model or vacuum boundary conditions. The contributions to the reaction field by the covalently bound nearest-neighbour atoms that are excluded from the non-bonded van der Waals and Coulomb interaction were considered in all simulations.

\section{Simulations in atomistic water}

The four energy-minimized proteins were solvated in a cubic box with a minimum distance to the wall of $1.4 \mathrm{~nm}$, i.e. HEWL in 14,332 water molecules, CspA in 8,128 , GP in 7,444 , and $\mathrm{CM}$ in 20,643 , and simulated with periodic boundary conditions. The solvent was energy-minimized with the protein kept fixed by restraining all solute atoms to their positions in the starting structure through a harmonic potential energy term with a force constant of $2.5 \times 10^{4} \mathrm{~kJ} \mathrm{~mol}^{-1} \mathrm{~nm}^{-2}$. Sodium or chlorine counter ions were added to neutralize the total charge of the protein. Initial velocities were randomly generated from a Maxwell-Boltzmann distribution at $60 \mathrm{~K}$. During the equilibration, the temperature was increased stepwise from 60 to $300 \mathrm{~K}$ in steps of $60 \mathrm{~K}$, with a step size of $20 \mathrm{ps}$. The protein position restraints were simultaneously relaxed by lowering the force constant by a factor 10 at each step. The final configurations 
were used as starting structures for the 20-ns production runs. For the non-bonded interactions, a twin cutoff method was used with a short-range cutoff radius of $0.8 \mathrm{~nm}$, an intermediate-range cutoff radius of $1.4 \mathrm{~nm}$, and an update frequency of five time steps for the short-range pairlist and intermediaterange interactions. The $\mathrm{O}-\mathrm{H}$ and $\mathrm{H}-\mathrm{H}$ bond lengths in the solvent were constrained to the ideal SPC values applying the SHAKE algorithm (Ryckaert et al. 1977).

\section{Simulations in coarse-grained water}

The four proteins were solvated in a cubic box with a minimum distance to the wall of 1.4-1.6 nm, i.e. HEWL in 3,217 CG beads, CspA in 1,659, GP in 1,669, and CM in 3,939, and simulated with periodic boundary conditions. The larger minimum distance to the wall is required by the larger non-bonded cutoff used in the mixed-grained simulations (see below). As starting structures, the equilibrated structures of the proteins from the simulations in FG water were used. The $\mathrm{CG}$ water was energy-minimized and subsequently equilibrated in three steps. In the first $100 \mathrm{ps}$, the protein atoms were positionally restrained with a force constant of $2.5 \times 10^{4} \mathrm{~kJ} \mathrm{~mol}^{-1} \mathrm{~nm}^{-2}$ and the volume was held constant. In the second step, a NPT simulation of 250 ps length was carried out with a positionrestraining force constant of $2.5 \times 10^{2} \mathrm{~kJ} \mathrm{~mol}^{-1} \mathrm{~nm}^{-2}$, and subsequently a 250 -ps simulation was performed without any position restraints. For the non-bonded interactions, a twin cutoff method was used with a short-range cutoff radius of $1.4 \mathrm{~nm}$, an intermediate-range cutoff radius of $2.0 \mathrm{~nm}$, and an update frequency of five time steps for the short-range pairlist and intermediate-range interactions.

\section{Simulations in implicit solvent and vacuum}

The four proteins were simulated using vacuum boundary conditions and the vacuum GROMOS force field 54B7 (Schmid et al. 2011). As starting structures, energy-minimised X-ray structures were used. The implicit solvent model (Fraternali and van Gunsteren 1996), which describes the force of the solvent on the protein atoms as a function of the solvent accessible surface area (SASA) of the protein, was used without volume correction (Allison et al. 2011). The model is described in detail in Refs. Fraternali and van Gunsteren (1996), Allison et al. (2011). For the non-bonded interactions, a twin cutoff method was used with a short-range cutoff radius of $0.8 \mathrm{~nm}$, an intermediate-range cutoff radius of $1.4 \mathrm{~nm}$, and an update frequency of five time steps for the short-range pairlist and intermediate-range interactions.

\section{Analysis}

The GROMOS analysis programs to calculate energies ene_ana, atom-positional root-mean-square deviations rmsd, atom-positional root-mean-square fluctuations rmsf, secondary structure elements $d s s p$, hydrogen bonds hbond, radii of gyration rgyr, torsional-angle transitions ditrans, NOE distance-bound violations prep_noe, noe and post_ noe, ${ }^{3} J$-coupling values jval, residual dipolar couplings svd_fit, and ${ }^{1} \mathrm{H}-{ }^{15} \mathrm{~N}$ order parameters nhoparam are described in more detail in Ref. Eichenberger et al. (2011).

\section{RMSD and RMSF}

The backbone atom-positional root-mean-square deviation (RMSD) between a configuration and a reference configuration has been calculated based on all backbone non-hydrogen atoms $\left(\mathrm{N}, \mathrm{C}_{\alpha}, \mathrm{C}, \mathrm{O}\right)$ using the following formula,

$\operatorname{RMSD}(\mathrm{bb})=\sqrt{\frac{1}{N_{\mathrm{bb}}} \sum_{i=1}^{N_{\mathrm{bb}}}\left(\mathbf{r}_{i}-\mathbf{r}_{i, \mathrm{ref}}\right)^{2}}$,

where $N_{\mathrm{bb}}$ is the number of backbone atoms considered, $\mathbf{r}_{i}$ the position of atom $i$, and $\mathbf{r}_{i \text {,ref }}$ the position of atom $i$ in the reference configuration. As reference configurations, the $\mathrm{X}$-ray structures of the proteins studied were used (Artymiuk et al. 1982; Schindelin et al. 1994; Gallagher et al. 1994; Okvist et al. 2006).

The atom-positional root-mean-square fluctuations (RMSF) were calculated for the $C_{\alpha}$ atoms of the backbone and for the last non-hydrogen atom of each side chain [C $\mathrm{C}_{\beta}$ (ALA), $\mathrm{N}_{\eta 1}$ (ARG), $\mathrm{N}_{\delta 2}$ (ASN), $\mathrm{O}_{\delta 1}$ (ASP), $\mathrm{S}_{\gamma}$ (CYS), $\mathrm{N}_{\varepsilon 2}$ (GLN), $\mathrm{O}_{\varepsilon 1}$ (GLU), $\mathrm{C}_{\alpha}$ (GLY), $\mathrm{C}_{\varepsilon 1}$ (HIS), $\mathrm{C}_{\delta}$ (ILE), $\mathrm{C}_{\delta 1}$ (LEU), $\mathrm{N}_{\zeta}$ (LYS), $\mathrm{C}_{\varepsilon}$ (MET), $\mathrm{C}_{\zeta}$ (PHE), $\mathrm{C}_{\gamma}$ (PRO), $\mathrm{O}_{\gamma}$ (SER), $\mathrm{O}_{\gamma 1}$ (THR), $\mathrm{C}_{\zeta 2}$ (TRP), $\mathrm{O}_{\eta}$ (TYR), $\mathrm{C}_{\gamma 1}$ (VAL)] using the following formula,

$\operatorname{RMSF}_{i}=\sqrt{\frac{1}{N_{t}} \sum_{t=1}^{N_{t}}\left(\mathbf{r}_{i}(t)-\left\langle\mathbf{r}_{i}\right\rangle\right)^{2}}$,

where $i$ indicates the $\mathrm{C}_{\alpha}$ or side-chain atom of residue $i,\left\langle\mathbf{r}_{i}\right\rangle$ its average position, and $N_{t}$ is the number of configurations in the simulation. The RMSF was further averaged over all residues using a mean-square sum,

$\langle\mathrm{RMSF}\rangle_{x}=\sqrt{\frac{1}{N_{i}} \sum_{i=1}^{N_{i}}\left(\mathrm{RMSF}_{x, i}\right)^{2}}$,

where $x$ indicates the backbone $\left(\mathrm{C}_{\alpha}\right)$ or side chain atoms (sc), and $N_{i}$ is the number of residues.

\section{Radius of gyration}

The radius of gyration of a protein is a measure of the compactness of a structure and can be related to the lightscattering intensity, 
$R_{\mathrm{gyr}}=\sqrt{\frac{1}{N_{a}} \sum_{i=1}^{N_{a}}\left(\mathbf{r}_{i}-\mathbf{R}_{\mathrm{com}}\right)^{2}}$

with

$\mathbf{R}_{\mathrm{com}}=\frac{1}{M} \sum_{i=1}^{N_{a}} m_{i} \mathbf{r}_{i}$

where $N_{a}$ is the number of protein atoms, $\mathbf{r}_{i}$ the position of atom $i, m_{i}$ the mass of atom $i$, and $M$ the total mass of the protein atoms.

\section{Secondary structure elements}

The secondary structure elements of the four proteins were detected and monitored based on the rules of Kabsch and Sander (1983). In order to avoid ambiguous assignment if a residue is part of two different secondary structure elements, the following priority rules were applied: $\beta$-strand $/ \beta$-bridge $>$ $\alpha$-helix $>\pi$-helix $>3_{10}$-helix $>$ hydrogen bonded turn $>$ bend.

\section{Hydrogen bonds}

The geometric criterion for a hydrogen bond was a minimum donor-hydrogen-acceptor angle of $135^{\circ}$ and a maximum hydrogen-acceptor distance of $0.25 \mathrm{~nm}$. The hydrogen bonds were split for each protein into three groups: backbonebackbone (bb-bb), backbone-side chain (bb-sc), and side chain-side chain (sc-sc). During a simulation, the number of configurations in which a hydrogen bond is present, $N_{\mathrm{hb}}$, is counted and the occurrence is given in percentage,

$\mathrm{occ}=100 \times \frac{1}{N_{f}} \sum_{t=1}^{N_{f}} N_{\mathrm{hb}}(t)$,

where $N_{f}$ is the total number of configurations in a simulation trajectory. For each of the three groups bb-bb, bb-sc and sc-sc, the occurrence of the corresponding hydrogen bonds was summed up.

\section{Torsional-angle transitions}

The $60^{\circ}$ and $120^{\circ}$-transitions of the torsional angles $\phi, \psi, \chi_{1}, \chi_{2}$, and $\chi_{3}$ were counted during the 20-ns simulation time. The root-mean-square fluctuations (RMSF) of the torsional angles were calculated and averaged analogously to Eqs. (2) and (3).

\section{${ }^{1} \mathrm{H}_{-}{ }^{15} \mathrm{~N}$ order parameters}

The ${ }^{1} \mathrm{H}-{ }^{15} \mathrm{~N}$ order parameters $\left(S^{2}\right)$ of hen egg-white lysozyme (HEWL) were calculated using the following equation (Henry and Szabo 1985; Chandrasekhar et al. 1992),
$S^{2}=\frac{1}{2}\left[3 \sum_{\alpha=1}^{3} \sum_{\beta=1}^{3}\left\langle\mu_{\alpha} \mu_{\beta}\right\rangle^{2}-1\right]$,

where $\mu_{\alpha}$ are $x-, y$-, and $z$-components of the normalised inter-atomic $\mathrm{N}-\mathrm{H}$ vector. The order parameters were compared to experimental data of HEWL (Buck et al. 1995).

NMR NOE proton-proton distances and ${ }^{3} J$-coupling constants

A total of 1,630 experimental NMR proton-proton upper distance bounds of HEWL were taken from Ref. Schwalbe et al. (2001) and pseudo-atom distance corrections as given by Wüthrich et al. (1983) were added. The proton-proton distances in the simulation were calculated using $1 / r^{6}$ averaging, i.e. $\left\langle r^{-6}\right\rangle^{-\frac{1}{6}}$. Experimental ${ }^{3} J$-coupling constants of HEWL reported by Smith et al. (1991) were used for comparison to calculated coupling constants.

Backbone ${ }^{15} \mathrm{~N}-{ }^{1} \mathrm{H},{ }^{13} \mathrm{C}_{\alpha^{-}}{ }^{13} \mathrm{C}$, and ${ }^{13} \mathrm{C}^{15}{ }^{15} \mathrm{~N}$ residual dipolar couplings

The residual dipolar coupling (RDC) $D_{i j}$ between two spins $i$ and $j$ is given by

$D_{i j}=-\frac{\gamma_{i} \gamma_{j} \mu_{0} h}{8 \pi^{3}}\left\langle\frac{P_{2}\left(\cos \left(\theta_{i j}\right)\right)}{r_{i j}^{3}}\right\rangle$,

where $\gamma_{i}$ is the gyromagnetic ratio of spin $i, \mu_{0}$ the magnetic permittivity of vacuum, $h$ Planck's constant, $P_{2}$ the second-order Legendre polynomial, and $\theta_{i j}$ the angle between the inter-nuclear vector $\mathbf{r}_{i j}$ and the static magnetic field. If the protein is more or less rigid, Eq. (8) can be reformulated such that the averaging over different orientations of the protein with respect to the magnetic field is represented by an alignment tensor $\underline{\mathbf{A}}$,

$D_{i j}=-\frac{\gamma_{i} \gamma_{j} \mu_{0} h}{8 \pi^{3}} \sum_{k \in x, y, z} \sum_{l \in x, y, z} \underline{A}_{k l} \cos \left(\zeta_{k}\right) \cos \left(\zeta_{l}\right)$,

where $\zeta_{x}, \zeta_{y}$ and $\zeta_{z}$ are the angles between the inter-nuclear vector and the $x$-, $y$-, and $z$-axes, respectively. The alignment tensor $\underline{\mathbf{A}}$ is determined for every configuration in the trajectory by a singular-value decomposition (SVD), solving the equation

$\underline{\mathbf{C}} \underline{\mathbf{a}}=\underline{\mathbf{R}}$,

where the matrix a contains the five independent elements of the $3 \times 3$ alignment tensor, and the matrix $\underline{\mathbf{R}}$ contains the $N_{R D C}$ experimental RDCs used for the fit. The resulting $Q$-value reflects the quality of each SVD fit, 
$Q=\frac{\sqrt{\frac{1}{N_{\mathrm{RDC}}} \sum_{i=1}^{N_{\mathrm{RDC}}}\left(D_{i}^{\exp }-D_{i}^{\text {calc }}\right)^{2}}}{\sqrt{\frac{1}{N_{\mathrm{RDC}}} \sum_{i=1}^{N_{\mathrm{RDC}}}\left(D_{i}^{\exp }\right)^{2}}}$,

and can be displayed as a distribution for all configurations in the trajectory.

\section{Results and discussion}

First, the influence of the process of coarse graining on various non-measurable properties is analysed by a comparison of the configurational ensembles generated in the four different environments. The focus is on the FG and CG simulations, while the results of the SASA and vacuum simulations were added for completeness. Second, a comparison of simulated with measured values for quantities that can be measured or derived from experimental data is presented for HEWL, for which ample NMR data are available.

\section{Comparison of environments}

The four proteins hen egg-white lysozyme (HEWL), major cold shock protein (CspA), G-protein (GP), and chorismate mutase (CM) were simulated for $20 \mathrm{~ns}$ in four different environments, i.e. in FG water (SPC), in CG water, in implicit solvent (a SASA model), and in vacuo, and their structural and dynamic properties were analysed and compared. As reference thereby the simulation in FG water is used, although an atomic level simulation also has finite accuracy in regard to the reproduction of experimental data.

Atom-positional RMSD, RMSF, and radius of gyration

The atom-positional root-mean-square deviations (RMSD) of the backbone atoms with respect to the crystal structure are shown in Fig. 2. No clear trend can be observed. For HEWL and CM, the RMSD in CG water is closest to the RMSD in FG water. The protein in the implicit solvent and in vacuo deviates more from the crystal structure in the case of HEWL and less in the case of CM. For CspA, the RMSDs of the protein in CG water and in vacuo are closest to the RMSD of the protein in FG water. In the case of GP, all other environments have a larger RMSD compared to the protein in FG water, with the protein in CG water and in implicit solvent deviating the most.

The average atom-positional root-mean-square fluctuations (RMSF) over $20 \mathrm{~ns}$ of simulation of the backbone $C_{\alpha}$ atoms and of the last non-hydrogen atom of each side chain are given in Table 1. Again, no clear trend is observable. Both the average backbone RMSF and the average side-chain RMSF of the protein in CG water underestimate the ones in FG water in the case of HEWL, CspA, and CM, but overestimate them in the case of GP. The average RMSFs in implicit solvent and in vacuo are sometimes closer to the value in FG water than the value in $\mathrm{CG}$ water is, and sometimes further away.

The radius of gyration (RGYR) of the protein in CG water is similar to the one of the protein in FG water in the case of HEWL, CspA, and CM, and slightly increased in the case of GP (Fig. 3). In the latter case, the simulation in vacuo results in the most similar RGYR.

\section{Secondary structure elements, intra-protein hydrogen bonds and energies}

The time evolution of the secondary structure elements is shown in Fig. 4. Overall, the secondary structure of all four proteins is stable in CG water. In the case of HEWL, seven of the eight helices (residues 5-14, 20-22, 25-36, 80-84, 89-101, 109-114, and 120-123) and both $\beta$-strands (residues 43-45 and 51-53) reported in the crystal structure (Artymiuk et al. 1982) are maintained. Only the 310 -helix
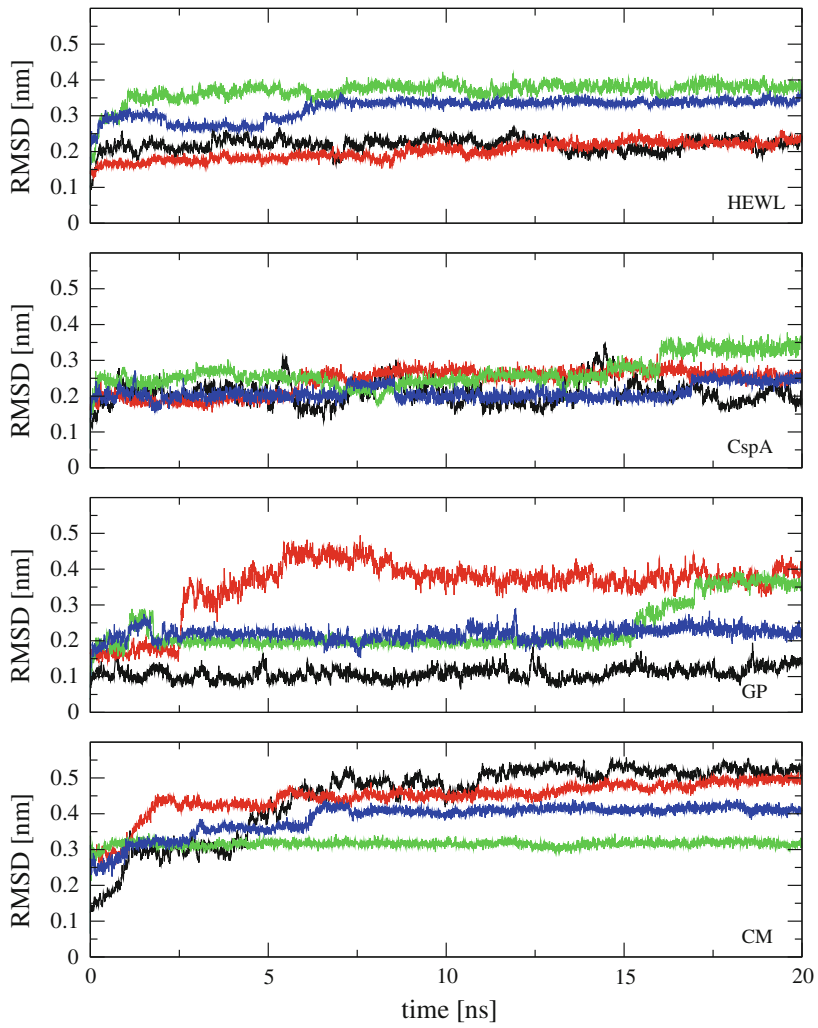

Fig. 2 Atom-positional root-mean-square deviations (RMSD) of the backbone with respect to the crystal structure of hen egg-white lysozyme (HEWL), major cold shock protein (CspA), G-protein (GP), and chorismate mutase (CM). Simulations in FG water are shown in black, those in CG water in red, those using the SASA model in green, and those in vacuum in blue 
Table 1 Average atom-positional root-mean-square fluctuation $\langle$ RMSF $\rangle$ of the backbone $\left(C_{\alpha}\right)$ and the last atom of the side chains (sc) using Eqs. (2) and (3) of hen egg-white lysozyme (HEWL), major cold shock protein (CspA), G-protein (GP), and chorismate mutase $(\mathrm{CM})$. All values in $\mathrm{nm}$ and calculated over $20 \mathrm{~ns}$

\begin{tabular}{|c|c|c|c|c|c|c|c|c|}
\hline \multirow[t]{2}{*}{ Solvent } & \multicolumn{2}{|c|}{ HEWL } & \multicolumn{2}{|l|}{ CspA } & \multicolumn{2}{|l|}{ GP } & \multicolumn{2}{|l|}{$\mathrm{CM}$} \\
\hline & $C_{\alpha}$ & $\mathrm{sc}$ & $C_{\alpha}$ & $\mathrm{sc}$ & $C_{\alpha}$ & $\mathrm{sc}$ & $C_{\alpha}$ & $\mathrm{sc}$ \\
\hline FG & 0.108 & 0.186 & 0.133 & 0.225 & 0.079 & 0.170 & 0.205 & 0.258 \\
\hline CG & 0.096 & 0.153 & 0.091 & 0.141 & 0.148 & 0.205 & 0.122 & 0.180 \\
\hline SASA & 0.105 & 0.179 & 0.132 & 0.131 & 0.221 & 0.217 & 0.093 & 0.167 \\
\hline Vacuo & 0.084 & 0.160 & 0.085 & 0.161 & 0.122 & 0.219 & 0.126 & 0.198 \\
\hline
\end{tabular}

(residues 104-107) is lost during the simulation. This helix is also lost in the simulation of the protein in vacuo together with the last helix (residues 120-123), which is lost in the implicit solvent simulation. The crystal structure (Schindelin et al. 1994) of CspA reports one helix (residues 35-37) and five $\beta$-strands (residues 5-13, 18-23, 30-34, 50-56, and 63-69), which are all maintained in the protein in CG water as well as in the other environments, although with more fluctuations. In the case of GP, one helix (residues 23-36) and four $\beta$-strands (residues 1-9, 12-20,
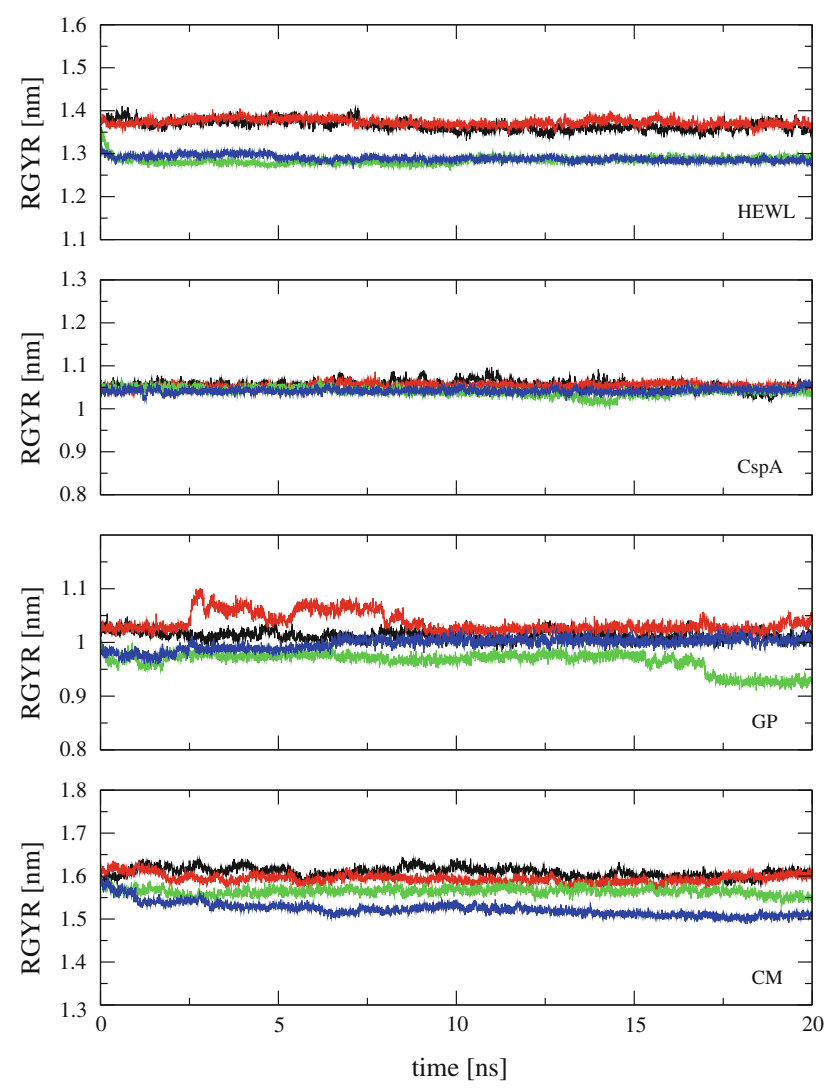

Fig. 3 Radius of gyration (RGYR) of hen egg-white lysozyme (HEWL), major cold shock protein (CspA), G-protein (GP), and chorismate mutase $(\mathrm{CM})$. Simulations in FG water are shown in black, those in CG water in red, those using the SASA model in green, and those in vacuum in blue
42-46 and 50-56) are reported in the crystal structure (Gallagher et al. 1994). One end of the helix unfolds partly during the simulation in CG water, and the third $\beta$-strand (residues 42-46) becomes thinner, while these secondary elements are completely stable in the protein in FG water. However, the same $\beta$-strand is also only partly stable in implicit solvent where in addition the second $\beta$-strand (residues 12-20) partly disappears. The secondary structure of CM consists of nine $\alpha$-helices (residues 3-16, 16-27, 33-51, 54-83, 84-87, 94-115, 115-120, 123-140, 142-154) as reported in the crystal structure (Okvist et al. 2006). These helices are maintained throughout the 20-ns simulation time in all environments.

The intra-protein hydrogen bonds (H-bonds) were split into backbone-backbone (bb-bb), backbone-side chain (bbsc), and side chain-side chain (sc-sc) H-bonds, and are listed in Table 2. Both the total number of H-bonds observed and the sum of the occurrence in percentage are given. In the case of bb-bb H-bonds, the total number observed in $\mathrm{CG}$ water is generally closer to the number in FG water compared to the other environments. In the simulations in vacuo, both the total number of $\mathrm{H}$-bonds and the summed occurrence are increased compared to FG water, indicating that the vacuum surrounding the protein favours these interactions. The same is found for the simulations in implicit solvent for HEWL and GP. In the case of CspA and CM, the total number of $\mathrm{H}$-bonds is increased in the implicit solvent but not the summed occurrence. The analysis of the bb-sc H-bonds shows that more and more stable H-bonds occur in all other environments compared to FG water, with the largest numbers of H-bonds found in $\mathrm{CG}$ water. In the case of sc-sc H-bonds, on the other hand, significantly more and more stable $\mathrm{H}$-bonds are observed in the protein in $\mathrm{CG}$ water compared to the three other environments. This is not entirely surprising given that the polar side chains at the surface of the protein can no longer form $\mathrm{H}$-bonds with the solvent and there is no additional term driving the polar side chains away from the protein as in the SASA model. In addition, the vacuum force field 54B7 used for the simulations in vacuum and in implicit solvent does not have full charges on polar side chains such 

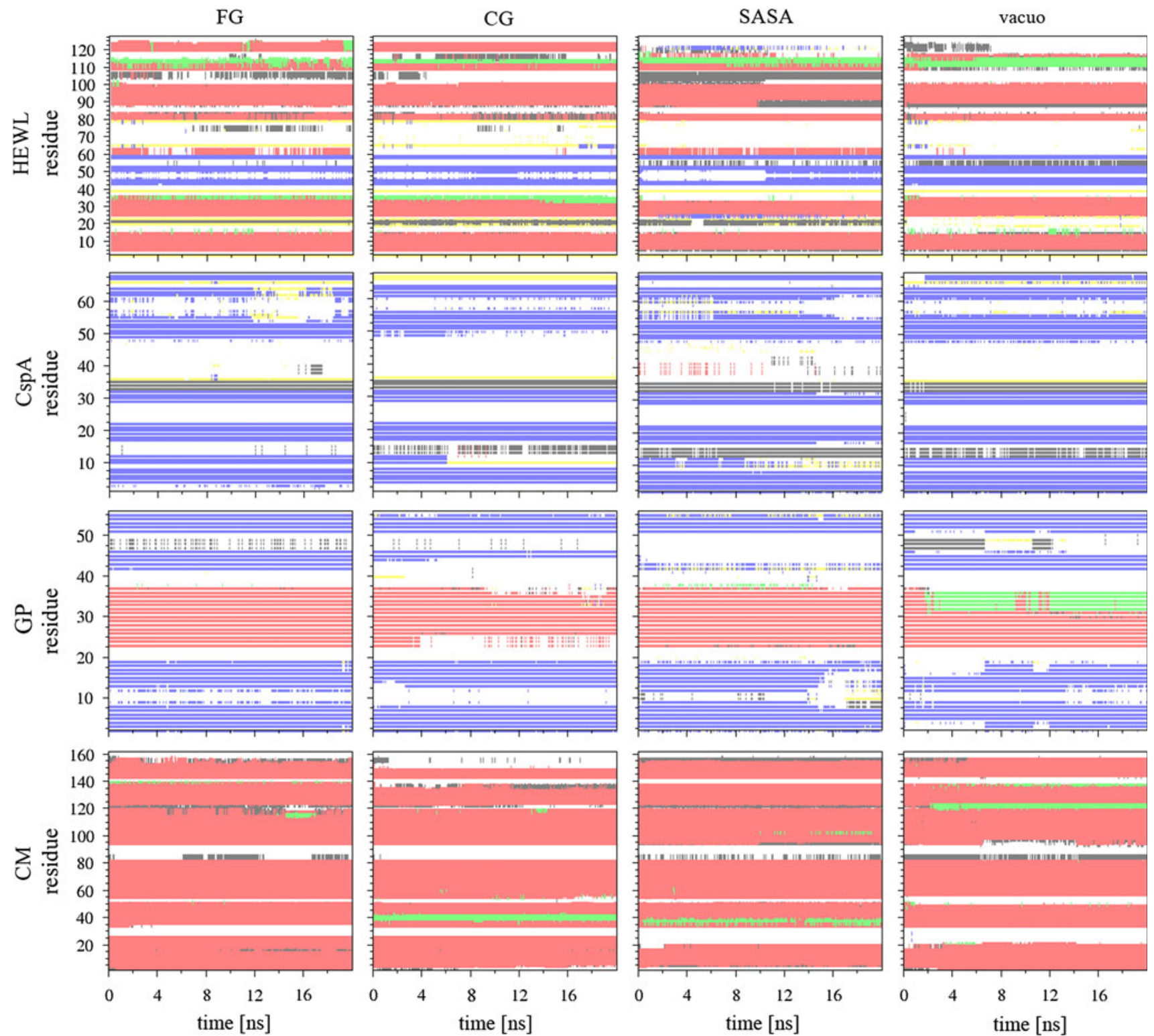

Fig. 4 From top to bottom, secondary structure elements of hen egg-white lysozyme (HEWL), major cold shock protein (CspA), G-protein (GP), and chorismate mutase (CM). $\alpha$-Helices are shown in red, $3_{10}$-helices in black, $\pi$-helices in green, $\beta$-bridges in yellow, and $\beta$-strands in blue

as lysine, arginine, etc., which also results in a smaller driving force to form sc-sc hydrogen bonds.

The observation of an increased number of sc-sc $\mathrm{H}$-bonds is reflected in the intra-protein potential energy and its components are listed in Table 3. H-bonds are electrostatically highly favourable, but the close vicinity of the atoms involved leads to a repulsive Lennard-Jones energy contribution. For all four proteins, the electrostatic energy term $V_{\mathrm{CRF}}$ is thus lower and the Lennard-Jones energy term $V_{\mathrm{LJ}}$ higher in $\mathrm{CG}$ water, resulting in an overall lower potential energy compared to FG water. Interestingly, both the implicit solvent and the vacuum environment lead to a lower $V_{\mathrm{LJ}}$ and a higher $V_{\mathrm{CRF}}$ compared to FG water.

\section{Torsional-angle analysis}

The Ramachandran plots of the backbone torsional angles $\phi$ and $\psi$ are shown in Fig. 5. As was already observed in the secondary structure element analysis, the secondary structure is well preserved in the mixed-grained simulations, and thus the size and position of the maxima in the Ramachandran plots are maintained. In the implicit solvent and in vacuo, additional configurations with torsional 
Table 2 Total number (\#) and total occurrence (occ) over $20 \mathrm{~ns}$ of hydrogen bonds in the protein of hen egg-white lysozyme (HEWL), major cold shock protein (CspA), G-protein (GP), and chorismate mutase (CM), split into backbone-backbone (bb-bb), backbone-side chains (bb-sc), and side chain-side chain (sc-sc) hydrogen bonds. The total occurrence is given as the sum of the occurrences of the individual hydrogen bonds obtained using Eq. (6)

\begin{tabular}{|c|c|c|c|c|c|c|c|}
\hline \multirow[t]{2}{*}{ Protein } & \multirow[t]{2}{*}{ Solvent } & \multicolumn{2}{|c|}{ bb-bb } & \multicolumn{2}{|c|}{ bb-sc } & \multicolumn{2}{|c|}{ sc-sc } \\
\hline & & \# & occ & \# & occ & \# & occ \\
\hline \multirow[t]{4}{*}{ HEWL } & FG & 89 & 5,518 & 67 & 2,023 & 36 & 1,312 \\
\hline & CG & 88 & 5,854 & 116 & 4,288 & 75 & 2,766 \\
\hline & SASA & 102 & 6,050 & 73 & 2,805 & 34 & 1,629 \\
\hline & Vacuo & 101 & 6,634 & 78 & 3,299 & 33 & 1,477 \\
\hline \multirow[t]{4}{*}{ CspA } & FG & 43 & 2,967 & 13 & 468 & 22 & 573 \\
\hline & CG & 46 & 2,953 & 59 & 1,640 & 51 & 1,663 \\
\hline & SASA & 48 & 2,765 & 25 & 1,137 & 8 & 385 \\
\hline & Vacuo & 53 & 3,782 & 26 & 1,080 & 13 & 603 \\
\hline \multirow[t]{4}{*}{ GP } & FG & 36 & 2,938 & 20 & 570 & 16 & 557 \\
\hline & CG & 40 & 2,381 & 44 & 1,393 & 64 & 1,689 \\
\hline & SASA & 62 & 3,616 & 26 & 1,140 & 14 & 548 \\
\hline & Vacuo & 55 & 2,918 & 34 & 1,015 & 17 & 548 \\
\hline \multirow[t]{4}{*}{$\mathrm{CM}$} & FG & 124 & 9,404 & 43 & 1,206 & 47 & 1,477 \\
\hline & CG & 143 & 9,246 & 92 & 3,438 & 153 & 5,217 \\
\hline & SASA & 141 & 9,359 & 80 & 3,213 & 42 & 1,415 \\
\hline & Vacuo & 158 & 9,968 & 82 & 2,899 & 50 & 1,775 \\
\hline
\end{tabular}

Table 3 Internal potential energy of the protein $V_{\text {pot }}$ and its components, the Lennard-Jones energy $V_{\mathrm{LJ}}$, and the electrostatic energy $V_{\mathrm{CRF}}$ of hen egg-white lysozyme (HEWL), major cold shock protein (CspA), G-protein (GP), and chorismate mutase (CM)

\begin{tabular}{llllc}
\hline Protein & Solvent & $\begin{array}{l}V_{\text {pot }} \\
\left(\mathrm{kJ} \mathrm{mol}^{-1}\right)\end{array}$ & $\begin{array}{l}V_{\mathrm{LJ}} \\
\left(\mathrm{kJ} \mathrm{mol}^{-1}\right)\end{array}$ & $\begin{array}{l}V_{\mathrm{CRF}} \\
(\mathrm{kJ} \mathrm{mol}\end{array}$ \\
\hline HEWL & FG & $-14,385$ & $-3,417$ & $-10,967$ \\
& CG & $-16,884$ & $-3,146$ & $-13,738$ \\
& SASA & $-13,434$ & $-3,539$ & $-9,895$ \\
& Vacuo & $-13,643$ & $-3,603$ & $-10,040$ \\
CspA & FG & $-6,154$ & $-1,621$ & $-4,533$ \\
& CG & $-8,406$ & $-1,403$ & $-7,003$ \\
& SASA & $-4,160$ & $-1,664$ & $-2,496$ \\
& Vacuo & $-4,211$ & $-1,728$ & $-2,483$ \\
GP & FG & $-6,275$ & $-1,375$ & $-4,899$ \\
& CG & $-8,253$ & $-1,099$ & $-7,154$ \\
& SASA & $-3,672$ & $-1,373$ & $-2,299$ \\
& Vacuo & $-3,762$ & $-1,446$ & $-2,316$ \\
CM & FG & $-17,701$ & $-4,256$ & $-13,446$ \\
& CG & $-22,253$ & $-3,902$ & $-18,352$ \\
& SASA & $-15,270$ & $-4,610$ & $-10,659$ \\
& Vacuo & $-15,730$ & $-4,687$ & $-11,043$ \\
\hline & & & &
\end{tabular}

angles in the upper right quarter were sampled in all four proteins compared to the protein in FG water.

The total number of transitions of the backbone torsional angles $\phi$ and $\psi$ and of the side-chain torsional angles $\chi_{1}, \chi_{2}$ and $\chi_{3}$ are listed together with the respective average rootmean-square fluctuation in Table 4. For the backbone angles, the total number of transitions observed in the protein in $\mathrm{CG}$ water is closest to the value of the protein in FG water (except for $\phi$ in CM and $\psi$ in CspA). There is no clear trend in the average RMSF values but they are generally close between all environments with a few exceptions, e.g. the RMSF of $\phi$ in CspA and of $\psi$ in GP in the vacuum environment. The same general trend holds for the first two side-chain torsional angles $\chi_{1}$ and $\chi_{2}$, although the average RMSF of $\chi_{2}$ is generally lower in CG water than in the other environments. This is even more pronounced for $\chi_{3}$, where the average RMSF in CG water is significantly lower and also the total number of transitions is smaller for all four proteins. This finding is in agreement with the increased number of hydrogen bonds involving side chains. There are only five amino acids with a $\chi_{3}$ torsional angle and four of these five amino acids are polar ones forming hydrogen bonds, thus dominating the analysis for this torsional angle.

\section{Comparison to experiment}

The simulations of hen egg-white lysozyme (HEWL) in FG water, in $\mathrm{CG}$ water, and in vacuo were compared to experimental data, i.e. NOE distance bounds, ${ }^{1} \mathrm{H}-{ }^{15} \mathrm{~N}$ order parameters, ${ }^{3} J_{H_{N} H_{\alpha}}$-coupling constants, and residual dipolar couplings (RDC).

The distributions of the NOE distance bound violations observed in the three simulations are shown in Fig. 6. In general, the number and size of the violations are slightly increased in the mixed-grained simulation but smaller compared to the vacuum environment. There were six violations larger than $0.3 \mathrm{~nm}$ found in the protein in FG water, which are, however, not crucial as all of them involve the carboxy terminal residue (LEU129), which is very mobile. In the protein in CG water, also six violations larger than $0.3 \mathrm{~nm}$ were found and they also involve LEU129. In the vacuum simulation, 10 violations larger than $0.3 \mathrm{~nm}$ were observed. One of them involves LEU129 and three involve TRP28. The other six violations are between residues in different parts of the protein.

The ${ }^{1} \mathrm{H}-{ }^{15} \mathrm{~N}$ order parameters of the protein in the three environments are compared to order parameters derived from experiment in Fig. 7. The order parameters from the simulations are generally smaller than those derived from the experiment. The largest order parameters are found for residues in helices. This pattern is reproduced roughly by 

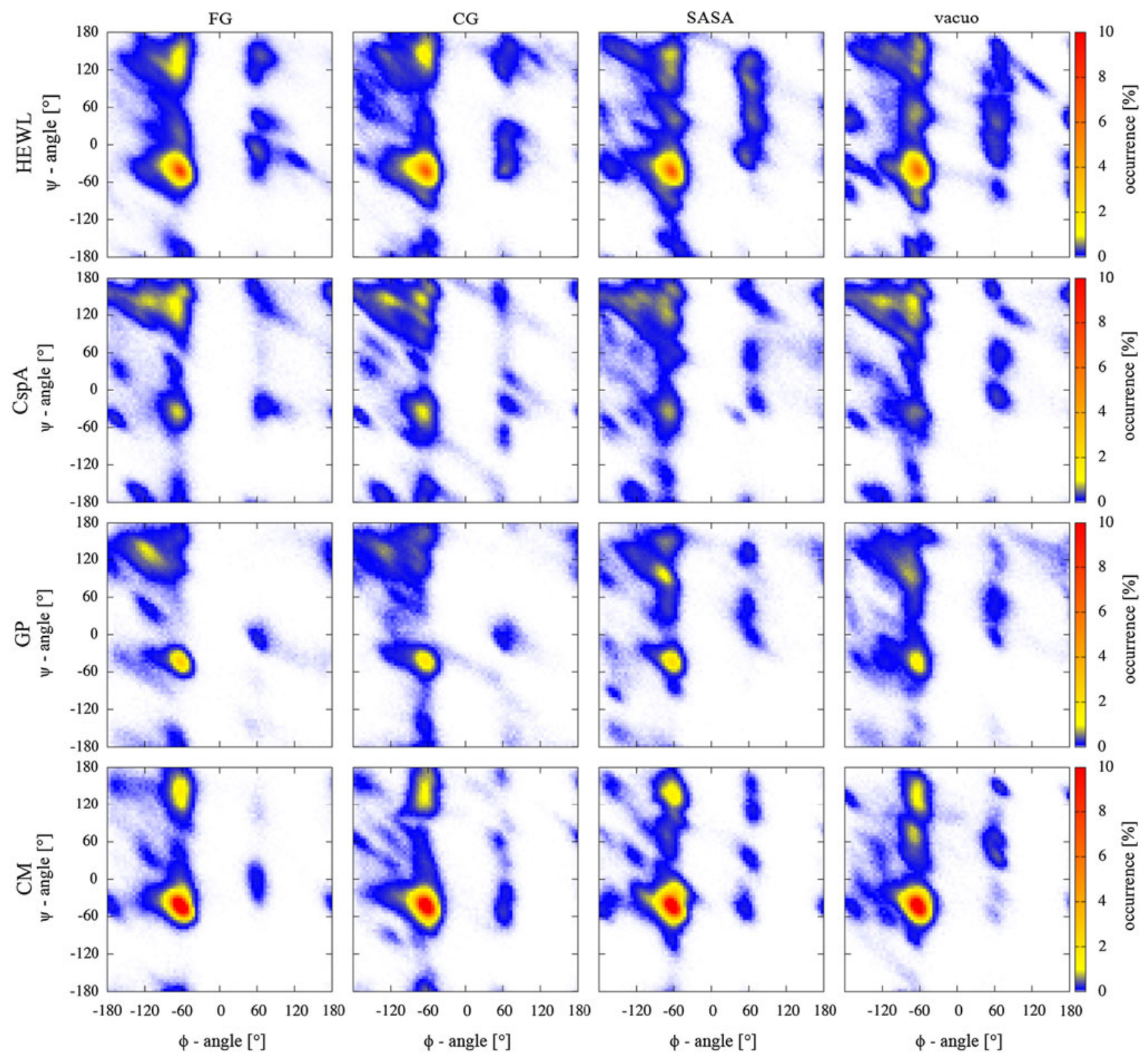

Fig. 5 From top to bottom, Ramachandran plots of hen egg-white lysozyme (HEWL), major cold shock protein (CspA), G-protein (GP), and chorismate mutase $(\mathrm{CM})$

all three simulations. The vacuum simulation, however, largely overestimates the order parameter of residues 85 and $102 / 103$, while the CG simulation only overestimates the order parameter of residue 85 . The largest differences between the order parameters in the CG simulation compared to the FG simulation and the experimental data are observed for residues 74 and 102-105, for which the CG simulation yields much less order. Yet, the $C_{\alpha}$ positional RMS fluctuations for these residues display similar values, i.e. mobility (data not shown). The total number of bb-bb hydrogen bonds involving residues in the range 70-80 and $100-110$ is 25 for the FG simulation and 24 for the CG simulation. For the bb-sc hydrogen bonds, these numbers are 8 (FG) and $25(\mathrm{CG})$, and for the sc-sc hydrogen bonds these numbers are $6(\mathrm{FG})$ and $18(\mathrm{CG})$. A detailed comparison of these hydrogen bonds with high occurrence, i.e. $\geq 50 \%$, is given in Table 5. The bb-bb hydrogen bond patterns in the range $70-80$ for the FG and CG simulations are similar. In the range of 100-110, the patterns are different, e.g. the hydrogen bond between residue 105 and 108 is very stable in the FG simulation but barely present in the CG simulation while for the hydrogen bond between residue 105 and 107 the picture is inverted. The hydrogen bonds in the CG simulation are generally more stable, which would indicate more order instead of less. Taken this together, there is no clear explanation for the difference in 
Table 4 Total number (\#) and average root-mean-square fluctuation $\langle\mathrm{RMSF}\rangle$ (in degrees) of the torsional angles $\phi, \psi, \chi_{1}, \chi_{2}$, and $\chi_{3}$ using Eqs. (2) and (3) of hen egg-white lysozyme (HEWL), major cold shock protein (CspA), G-protein (GP), and chorismate mutase (CM)

\begin{tabular}{|c|c|c|c|c|c|c|c|c|c|}
\hline \multirow[t]{2}{*}{ Angle } & \multirow[t]{2}{*}{ Solvent } & \multicolumn{2}{|l|}{ HEWL } & \multicolumn{2}{|l|}{ CspA } & \multicolumn{2}{|l|}{ GP } & \multicolumn{2}{|l|}{$\mathrm{CM}$} \\
\hline & & \# & RMSF & $\#$ & RMSF & \# & RMSF & \# & RMSF \\
\hline \multirow[t]{4}{*}{$\phi$} & FG & 25,022 & 65 & 26,818 & 109 & 30,459 & 76 & 10,335 & 26 \\
\hline & CG & 35,464 & 77 & 22,973 & 79 & 23,624 & 76 & 15,389 & 31 \\
\hline & SASA & 17,366 & 72 & 32,426 & 95 & 12,989 & 76 & 13,055 & 44 \\
\hline & Vacuo & 19,588 & 81 & 29,703 & 140 & 18,676 & 77 & 11,864 & 42 \\
\hline \multirow[t]{4}{*}{$\psi$} & FG & 8,800 & 103 & 5,324 & 108 & 7,600 & 112 & 3,845 & 69 \\
\hline & CG & 10,232 & 92 & 9,381 & 101 & 11,167 & 108 & 8,670 & 81 \\
\hline & SASA & 19,571 & 94 & 11,452 & 124 & 19,444 & 96 & 10,191 & 70 \\
\hline & Vacuo & 15,267 & 104 & 11,767 & 105 & 16,237 & 282 & 10,865 & 73 \\
\hline \multirow[t]{4}{*}{$\chi_{1}$} & FG & 8,404 & 173 & 5,669 & 196 & 4,324 & 131 & 13,658 & 204 \\
\hline & CG & 9,433 & 158 & 4,496 & 147 & 4,759 & 146 & 23,057 & 175 \\
\hline & SASA & 13,136 & 152 & 13,051 & 203 & 10,844 & 205 & 20,497 & 169 \\
\hline & Vacuo & 10,908 & 144 & 8,422 & 160 & 9,001 & 165 & 19,383 & 152 \\
\hline \multirow[t]{4}{*}{$\chi_{2}$} & FG & 44,809 & 420 & 15,636 & 1,105 & 16,696 & 661 & 37,981 & 804 \\
\hline & CG & 47,733 & 304 & 16,640 & 322 & 16,661 & 347 & 43,480 & 360 \\
\hline & SASA & 46,484 & 719 & 26,517 & 2,396 & 23,550 & 1,953 & 57,734 & 1,165 \\
\hline & Vacuo & 42,776 & 848 & 21,773 & 1,078 & 21,779 & 817 & 50,065 & 974 \\
\hline \multirow[t]{4}{*}{$\chi_{3}$} & FG & 12,265 & 721 & 9,944 & 1,071 & 13,275 & 1,245 & 31,937 & 759 \\
\hline & CG & 10,075 & 414 & 6,916 & 230 & 8,384 & 360 & 28,440 & 452 \\
\hline & SASA & 13,664 & 1,354 & 12,607 & 1,442 & 18,499 & 2,805 & 37,196 & 1,782 \\
\hline & Vacuo & 12,332 & 541 & 11,857 & 1,668 & 16,120 & 1,087 & 37,387 & 1,368 \\
\hline
\end{tabular}

the order parameters. However, the different number and occurrence of the side chain hydrogen bonds may explain the loss of the $3_{10}$ helix (residues 104-107) observed in the CG simulation. Especially the high occurrence of a hydrogen bond between the side chains of residues 106 and 116 as well as between the side chain of residue 103 and the backbone of residue 21 leads to an unfolding of the helical structure at this location.

Experimental proton-proton ${ }^{3} J_{H_{N} H_{\alpha}}$-coupling constants of HEWL (Smith et al. 1991) were compared to calculated values from the simulations (Fig. 8). The root-mean-square deviations (RMSD) of the calculated coupling constants from the experimental values are $1.6,1.8$, and $1.8 \mathrm{~Hz}$ for HEWL in FG water, in CG water and in vacuo, respectively. However, the quality of the calculated ${ }^{3} J$-couplings is low because of the inaccuracy due to the empirical nature of the parameters of the Karplus relation, which is used to obtain a ${ }^{3} J$-coupling from a configuration (Allison and van Gunsteren 2009; Steiner et al. 2012). Additionally, the sampling in a simulation is limited compared to the experimental time scale.

The quality of fitting the backbone ${ }^{15} \mathrm{~N}-{ }^{1} \mathrm{H},{ }^{13} \mathrm{C}_{\alpha^{-}}{ }^{13} \mathrm{C}$, and ${ }^{13} \mathrm{C}-{ }^{15} \mathrm{~N}$ residual dipolar couplings (RDC) calculated from simulation to the experimental RDCs of HEWL (Higman 2004) is assessed by a so-called $Q$-value (Eq. 11).
The closer a $Q$-value is to zero, the better is the quality of the fit. The distribution of the $Q$-values observed in the three simulations is shown in Fig. 9. The values of the simulations are all much higher than the value calculated from the crystal structure (Artymiuk et al. 1982). The protein in FG water results in $Q$-values closest to zero compared to the other two environments. Interestingly, the mixed-grained simulation performs similarly to the purely atomistic simulation for the ${ }^{13} \mathrm{C}_{\alpha}-{ }^{13} \mathrm{C}$ RDCs and worse for the ${ }^{13} \mathrm{C}-{ }^{15} \mathrm{~N}$ RDCs, while the performance for the ${ }^{15} \mathrm{~N}-{ }^{1} \mathrm{H}$ RDCs is worse than in the vacuum simulation.

\section{Computational efficiency}

A simulation of 500 steps of HEWL in FG water, in CG water, and in implicit solvent was performed with a single CPU and with four CPUs using MPI or OpenMP parallelisation. The number of CG beads in the mixed-grained simulation represented the same number of water molecules as present in the purely atomistic simulation to allow a fair comparison. The simulation in CG water is an order of magnitude faster than the purely atomistic simulation independent of the number of CPUs used, although much larger cutoffs for all non-bonded interactions are used in the mixed-grained simulations, i.e. 1.4 and $2.0 \mathrm{~nm}$ versus 


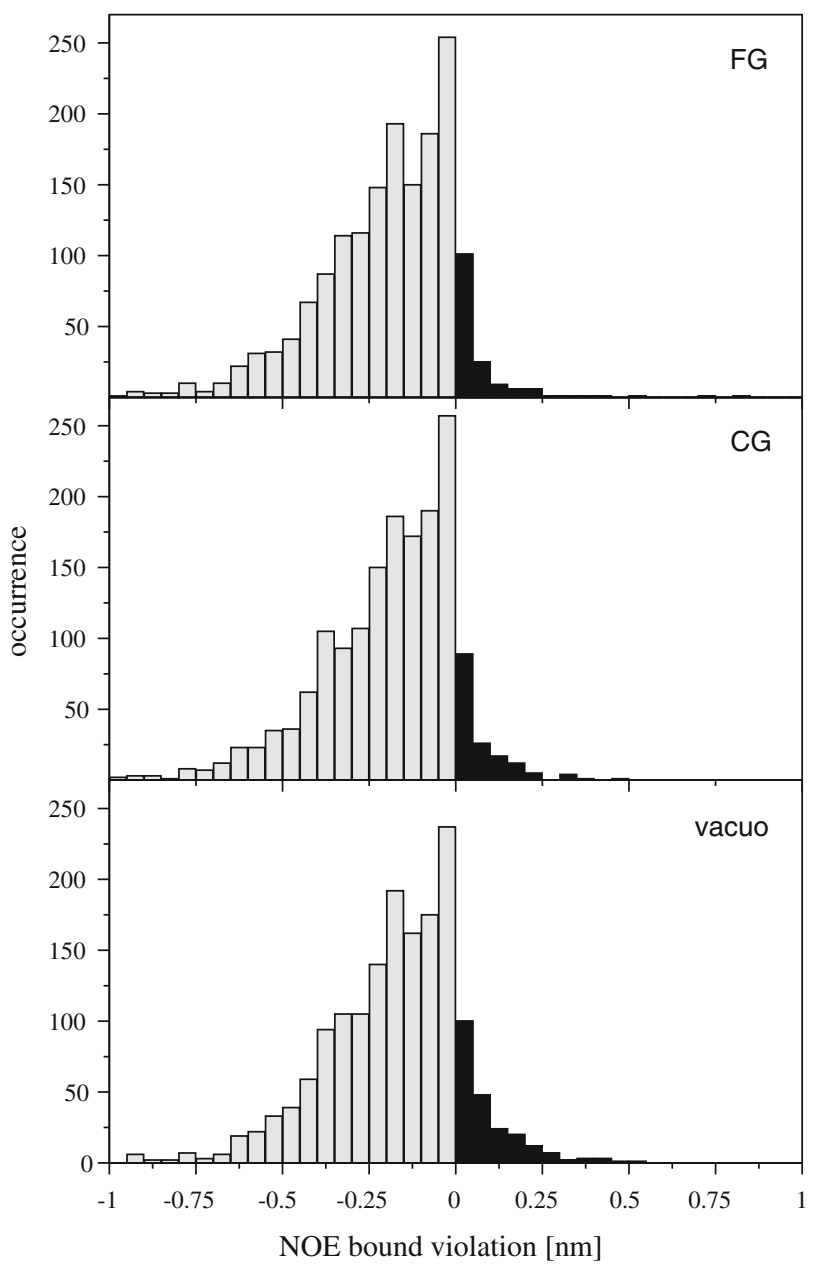

Fig. 6 Distribution of 1,630 experimental NOE-bound violations for the 20-ns simulation of hen egg-white lysozyme (HEWL) in FG water (top), CG water (middle), and in vacuo (bottom). NOE-bound violations are coloured black, while the satisfied NOE bounds appear in grey

0.8 and $1.4 \mathrm{~nm}$. The use of four instead of one CPU leads to a speed-up of a factor three. On a single CPU, the simulations in CG water and in implicit solvent are equally fast. However, the SASA model does not allow parallelisation as well as the CG model, resulting in a factor two difference in speed on four CPUs.

\section{Summary and conclusions}

Molecular dynamics (MD) simulations of four proteins, hen egg-white lysozyme (HEWL), major cold shock protein (CspA), G-protein (GP), and chorismate mutase (CM), solvated in coarse-grained (CG) water were analysed in terms of structural and dynamic properties, and compared to simulations of the same proteins in atomistic, finegrained (FG) water, in implicit solvent (SASA model), and in vacuo. In addition, the simulations of HEWL were

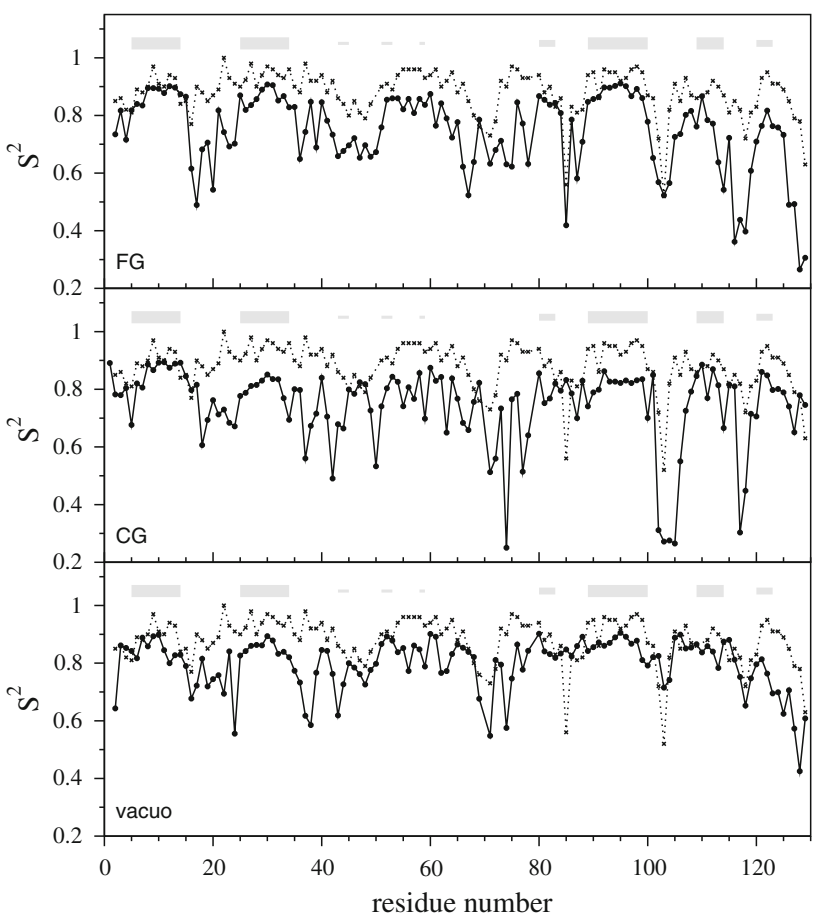

Fig. $7{ }^{1} \mathrm{H}-{ }^{15} \mathrm{~N}$ order parameters of hen egg-white lysozyme (HEWL) derived from experiment (Buck et al. 1995) (dotted line with crosses) compared to calculated order parameters (solid line with circles) of HEWL in FG water (top), CG water (middle), and in vacuum (bottom). ${ }^{1} \mathrm{H}-{ }^{15} \mathrm{~N}$ order parameters are calculated over the whole $20 \mathrm{~ns}$ of simulation time using a 1-ns time window for averaging. The grey bars on top of each graph indicate the secondary structure elements of HEWL, i.e. $\alpha$-helix (thick bars), $3_{10}$-helix (medium bars), and $\beta$-strand (thin bars)

compared to experimental NOE distance bounds, ${ }^{3} J$-coupling constants, $\mathrm{N}-\mathrm{H}$ order parameters, and backbone residual dipolar couplings.

The comparison of the backbone atom-positional rootmean-square deviation (RMSD) with respect to the crystal structures, the atom-positional root-mean-square fluctuations (RMSF) of the backbone and the side chains, the radius of gyration, the evolution of the secondary structure elements, and the Ramachandran plots shows no significant difference between the purely atomistic simulations and mixed-grained simulations. The protein in CG water generally performs better compared to the simulations in implicit solvent or in vacuo. The major difference observed is an increased occurrence of side chain-side chain (sc-sc) hydrogen bonds due to the lack of hydrogen-bond partners in the solvent. For the simulations in implicit solvent and in vacuo, the corresponding vacuum force field was used, which does not have full charges on the ARG, LYS, ASP, and GLU side chain and the polypeptide chain termini, therefore preventing the formation of salt bridges. In addition, the SASA model contains a term to drive polar side chains on the surface away from the protein. The 
Table 5 Details of the backbone - side chain (bb-sc) and side chainside chain (sc-sc) hydrogen bonds involving residues $70-80$ or $100-110$ of hen egg-white lysozyme (HEWL) observed in the simulations with FG water and CG water with an occurrence of at least 50 $\%$ in at least one of the simulations

\begin{tabular}{|c|c|c|c|c|c|}
\hline \multirow{2}{*}{$\begin{array}{l}\text { Donor } \\
\text { residue }\end{array}$} & \multirow{2}{*}{$\begin{array}{l}\text { Acceptor } \\
\text { residue }\end{array}$} & \multirow{2}{*}{$\begin{array}{l}\text { Donor } \\
\text { atoms }\end{array}$} & \multirow{2}{*}{$\begin{array}{l}\text { Acceptor } \\
\text { atom }\end{array}$} & \multicolumn{2}{|c|}{ Occurrence $[\%]$} \\
\hline & & & & FG & CG \\
\hline 65 ASN & 78 ILE & $\mathrm{N}-\mathrm{H}$ & $\mathrm{O}$ & 63 & 65 \\
\hline 73 ARG & $61 \mathrm{ARG}$ & $\mathrm{N}-\mathrm{H}$ & $\mathrm{O}$ & 70 & 86 \\
\hline 76 CYS & 63 TRP & $\mathrm{N}-\mathrm{H}$ & $\mathrm{O}$ & 94 & 93 \\
\hline 77 ASN & 74 ASN & $\mathrm{N}-\mathrm{H}$ & $\mathrm{O}$ & 54 & 79 \\
\hline 78 ILE & 76 CYS & $\mathrm{N}-\mathrm{H}$ & $\mathrm{O}$ & 77 & 80 \\
\hline $80 \mathrm{CYS}$ & 65 ASN & $\mathrm{N}-\mathrm{H}$ & $\mathrm{O}$ & 94 & 99 \\
\hline 82 ALA & 79 PRO & $\mathrm{N}-\mathrm{H}$ & $\mathrm{O}$ & 56 & 59 \\
\hline 83 LEU & 80 CYS & $\mathrm{N}-\mathrm{H}$ & $\mathrm{O}$ & 61 & 65 \\
\hline 100 SER & 96 LYS & $\mathrm{N}-\mathrm{H}$ & $\mathrm{O}$ & 81 & 38 \\
\hline 101 ASP & 97 LYS & $\mathrm{N}-\mathrm{H}$ & $\mathrm{O}$ & 37 & 96 \\
\hline 102 GLY & 98 ILE & $\mathrm{N}-\mathrm{H}$ & $\mathrm{O}$ & 1 & 61 \\
\hline 106 ASN & 104 GLY & $\mathrm{N}-\mathrm{H}$ & $\mathrm{O}$ & 37 & 70 \\
\hline 107 ALA & 105 MET & $\mathrm{N}-\mathrm{H}$ & $\mathrm{O}$ & 0 & 61 \\
\hline 108 TRP & 105 MET & $\mathrm{N}-\mathrm{H}$ & $\mathrm{O}$ & 90 & 2 \\
\hline 112 TRP & 108 TRP & $\mathrm{N}-\mathrm{H}$ & $\mathrm{O}$ & 65 & 96 \\
\hline 113 ASN & $109 \mathrm{VAL}$ & $\mathrm{N}-\mathrm{H}$ & $\mathrm{O}$ & 56 & 87 \\
\hline 115 CYS & 110 ALA & $\mathrm{N}-\mathrm{H}$ & $\mathrm{O}$ & 83 & 99 \\
\hline 67 GLY & 72 SER & $\mathrm{N}-\mathrm{H}$ & OG & 3 & 71 \\
\hline 72 SER & 60 SER & OG-HG & $\mathrm{O}$ & 41 & 58 \\
\hline 100 SER & 96 LYS & $\mathrm{OG}-\mathrm{HG}$ & $\mathrm{O}$ & 8 & 88 \\
\hline 103 ASN & $21 \mathrm{ARG}$ & ND2-HD2 & $\mathrm{O}$ & 0 & 57 \\
\hline $105 \mathrm{MET}$ & 23 TYR & $\mathrm{N}-\mathrm{H}$ & $\mathrm{OH}$ & 60 & 33 \\
\hline 108 TRP & $56 \mathrm{LEU}$ & NE1-HE1 & $\mathrm{O}$ & 74 & 51 \\
\hline 110 ALA & $35 \mathrm{GLU}$ & $\mathrm{N}-\mathrm{H}$ & OE2 & 51 & 65 \\
\hline $112 \mathrm{ARG}$ & 107 ALA & NE-HE & $\mathrm{O}$ & 2 & 57 \\
\hline $112 \mathrm{ARG}$ & 107 ALA & NH2-HH2 & $\mathrm{O}$ & 0.4 & 55 \\
\hline 20 TYR & 100 SER & $\mathrm{OH}-\mathrm{HH}$ & OG & 28 & 92 \\
\hline 27 ASN & 105 MET & ND2-HD2 & SD & 59 & 23 \\
\hline 65 ASN & 74 ASN & ND2-HD2 & OD1 & 26 & 58 \\
\hline 97 LYS & 101 ASP & NZ-HZ & OD1 & 6 & 62 \\
\hline 97 LYS & 101 ASP & NZ-HZ & OD2 & 0.4 & 65 \\
\hline 116 LYS & $106 \mathrm{ASN}$ & NZ-HZ & OD1 & 0.3 & 78 \\
\hline
\end{tabular}

increased number of sc-sc hydrogen bonds in the mixedgrained simulations is reflected in the intra-protein potential energy $V_{\text {pot }}$ and its components. Hydrogen bonds are electrostatically favourable but due to the short distance between the involved atoms the Lennard-Jones energy is less negative, i.e. more repulsive. Thus, the Lennard-Jones energy $V_{\mathrm{LJ}}$ of the protein in CG water is less negative compared to that of the protein in FG water and the electrostatic energy $V_{\mathrm{CRF}}$ is more negative. Interestingly, the opposite trend is found for the protein in implicit solvent and in vacuo, where $V_{\mathrm{LJ}}$ is

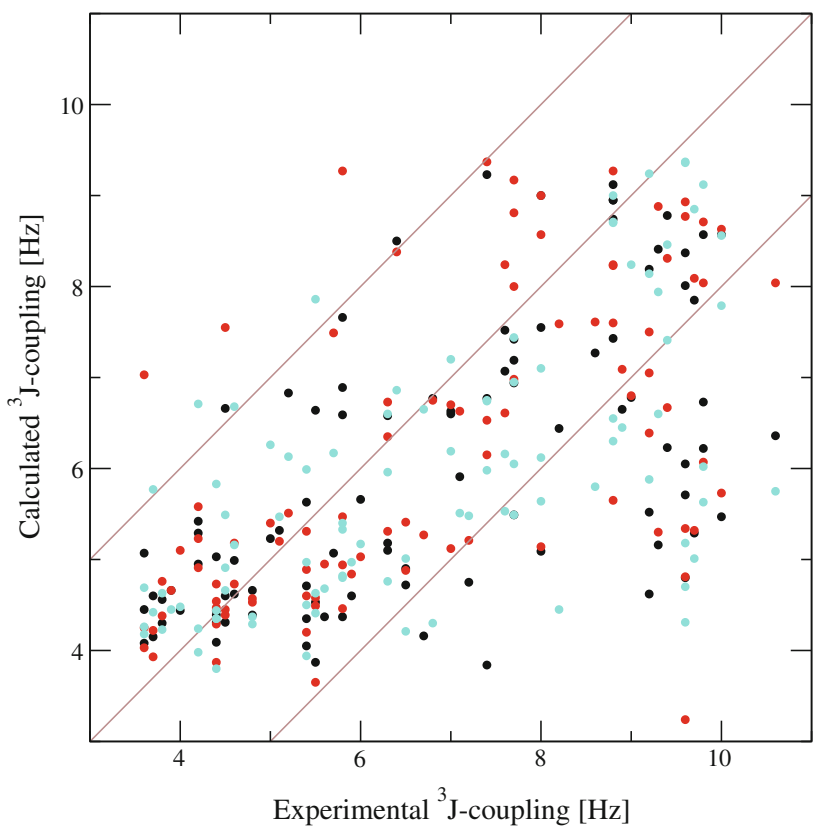

Fig. 8 Comparison of calculated ${ }^{3} J_{H_{N} H_{\alpha}}$-coupling constants of hen egg-white lysozyme (HEWL) in FG water (black), CG water (red), and in vacuum (cyan) to the experimental coupling constants of HEWL (Smith et al. 1991). The coupling constants were calculated from 20-ns simulation time. A 2-Hz region of uncertainty is indicated by brown lines (Steiner et al. 2012)

more negative and $V_{\mathrm{CRF}}$ is less negative compared to the protein in FG water. The effect of the larger occurrence of sc-sc hydrogen bonds can also be observed in the analysis of side-chain torsional angles $\chi_{1}, \chi_{2}$ and $\chi_{3}$. While the total number of transitions and the RMSF of the first two angles, which are present both in side chains forming hydrogen bonds (mostly polar) and in such not forming any (apolar), show no clear difference between the proteins in CG or FG water, significantly fewer transitions occur for the last angle $\chi_{3}$ and also the RMSF is much smaller in CG water compared to FG water. This angle occurs mainly in polar side chains. If these side chains form more and more stable hydrogen bonds, the number of transitions will decrease and thus the RMSF will be reduced.

Further analysis of the simulations of HEWL in FG water, in CG water, and in vacuo shows that the extent of agreement with the available experimental data is not much decreased in the mixed-grained simulation compared to the purely atomistic simulation. The number of NOE distance bound violations increases slightly and the RMSD of the calculated and measured ${ }^{3} J_{H_{N} H_{\alpha}}$-coupling constants increases from 1.6 to $1.8 \mathrm{~Hz}$. The distributions of the $Q$-values of the backbone RDCs are shifted but in general still smaller than in the vacuum simulation.

Finally, it can be stated that the fold of the atomistic proteins in CG water is stable within the 20 ns simulation 


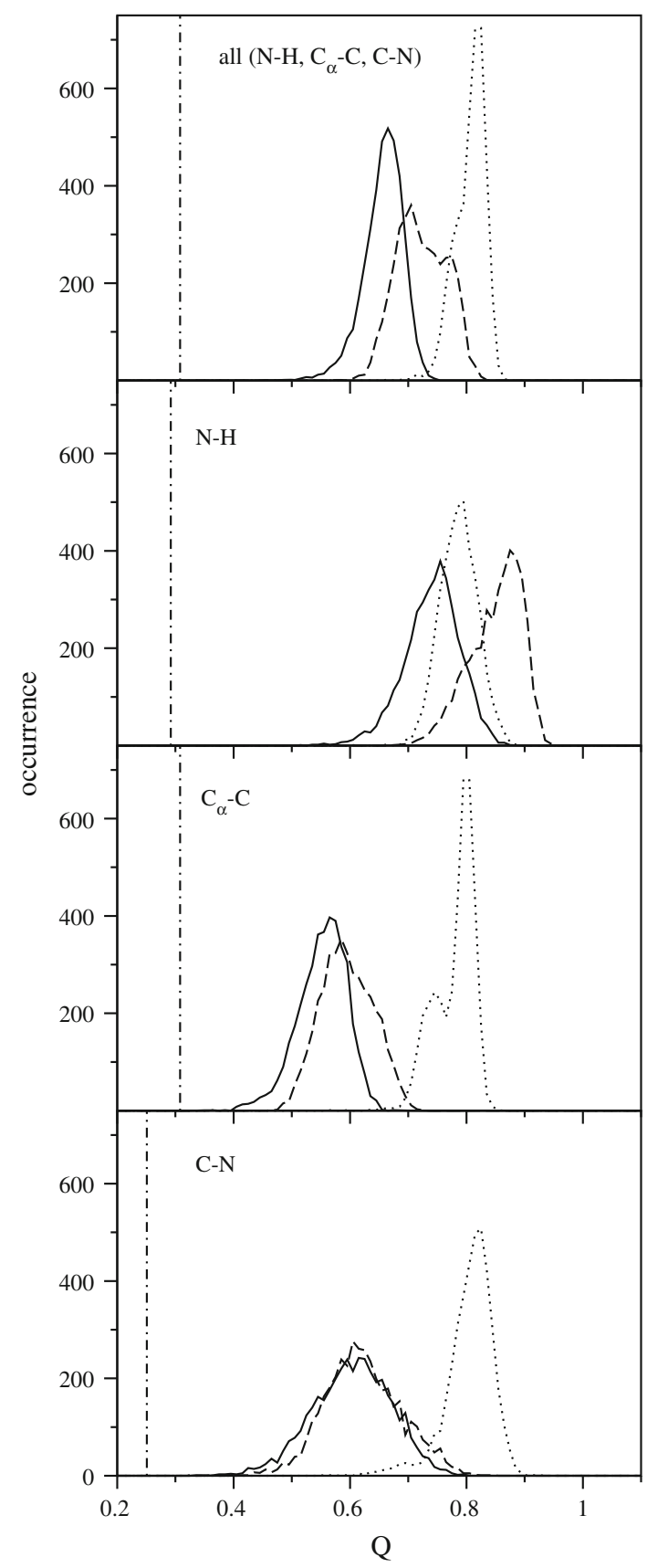

Fig. $9 Q$ value distribution of the calculated backbone $\mathrm{N}-\mathrm{H}, \mathrm{C}_{\alpha}-\mathrm{C}$, and $\mathrm{C}-\mathrm{N}$ RDCs of hen egg-white lysozyme (HEWL) in FG water (solid line), CG water (dashed line), and in vacuo (dotted line) when fitting the calculated residual dipolar couplings (RDC) of each configuration to the experimentally derived RDCs of HEWL (Higman 2004). The $Q$ value of the fits from the X-ray crystal structure (Artymiuk et al. 1982) is shown by a vertical dashed-dotted line

length and no larger alteration in the structural and dynamic properties can be detected except an increased occurrence of sc-sc hydrogen bonds. This difference comes, however, with a speed-up of an order of magnitude compared to purely atomistic simulations. Therefore, a possible application of these mixed-grained simulations can be in free energy difference calculations between a series of ligands bound to a protein, where only the overall stability of the secondary structure and thus of the (buried) binding pocket is important. The speed-up gained through the use of CG solvent would allow the application of accurate methods such as thermodynamic integration on larger data sets.

Acknowledgments This work was financially supported by the National Center of Competence in Research (NCCR) in Structural Biology and by grant number 200020-137827 of the Swiss National Science Foundation and by grant no. 228076 of the European Research Council, which is gratefully acknowledged.

\section{References}

Allison J, van Gunsteren WF (2009) A method to explore protein side chain conformational variability using experimental data. ChemPhysChem 10:3213-3228

Allison JR, Boguslawski K, Fraternali F, van Gunsteren WF (2011) A refined, efficient mean solvation force model that includes the interior volume contribution. J Phys Chem B 115:4547-4557

Artymiuk PJ, Blake CCF, Rice DW, Wilson KS (1982) The structures of the monoclinic and orthorhombic forms of hen egg-white lysozyme at 6 Angstroms resolution. Acta Crystallogr Sec B 38:778-783

Basdevant N, Ha-Duong T, Borgis D (2006) Particle-based implicit solvent model for biosimulations: application to proteins and nucleic acids hydration. J Chem Theory Comput 2:1646-1656

Berendsen HJC, Postma JPM, van Gunsteren WF, Hermans J (1981) Interaction models for water in relation to protein hydration. In: Pullmann B (ed) Intermolecular Forces. Reidel, Dordrecht, pp 331-342

Berendsen HJC, Postma JPM, van Gunsteren WF, DiNola A, Haak JR (1984) Molecular dynamics with coupling to an external bath. J Chem Phys 81:3684-3690

Buck M, Boyd J, Redfield C, MacKenzie DA, Jeenes DJ, Archer DB, Dobson CM (1995) Structural determinants of protein dynamics: analysis of ${ }^{15} \mathrm{~N}$ NMR relaxation measurements for main-chain and side-chain nuclei of hen egg white lysozyme. Biochemistry 34:4041-4055

Chandrasekhar I, Clore G, Szabo A, Gronenborn A, Brooks B (1992) A 500 ps molecular dynamics simulation study of interleukin- $1 \beta$ in water-correlation with nuclear magnetic resonance spectroscopy and crystallography. J Mol Biol 226:239-250

Eichenberger AP, Allison JR, Dolenc J, Geerke DP, Horta BAC, Meier K, Oostenbrink C, Schmid N, Steiner D, Wang D, van Gunsteren WF (2011) The GROMOS++ software for the analysis of biomolecular simulation trajectories. J Chem Theory Comp 7:3379-3390

Fraternali F, van Gunsteren WF (1996) An efficient mean solvation force model for use in molecular dynamics simulations of proteins in aqueous solution. J Mol Biol 256:939-948

Gallagher T, Alexander P, Bryan P, Gilliland GL (1994) Two crystal structures of the B1 immunoglobulin-binding domain of streptococcal protein $\mathrm{G}$ and comparison with NMR. Biochemistry 33:4721-4729

Henry E, Szabo A (1985) Influence of vibrational motion on solid state line shapes and NMR relaxation. J Chem Phys 82:4753-4761

Higman VA (2004) The use of bicelles and other ordered media to study protein structure and dynamics. Ph.D. thesis, University of Oxford 
Hockney RW (1970) The potential calculation and some applications. Methods Comput Phys 9:136-211

Kabsch W, Sander C (1983) Dictionary of protein secondary structure: pattern recognition of hydrogen-bonded and geometrical features. Biopolymers 22:2577-2637

Kunz A-PE, Allison JR, Geerke DP, Horta BAC, Hünenberger PH, Riniker S, Schmid N, van Gunsteren WF (2012) New functionalities in the GROMOS biomolecular simulation software. J Comput Chem 33:340-353

Lide DR (2007-2008) Handbook of chemistry and physics, 88th edn. CRC Press/Taylor and Francis, Boca Raton

Liu Y, Ichiye T (1996) Soft sticky dipole potential for liquid water: a new model. J Phys Chem 100:2723-2730

Masella M, Borgis D, Cuniasse P (2008) Combining a polarizable force-field and a coarse-grained polarizable solvent model: application to long dynamics simulations of bovine pancreatic trypsin inhibitor. J Comput Chem 29:1707-1724

Masella M, Borgis D, Cuniasse P (2011) Combining a polarizable forcefield and a coarse-grained polarizable solvent model. II. Accounting for hydrophobic effects. J Comput Chem 32:2664-2678

Michel J, Orsi M, Essex JW (2008) Prediction of partition coefficients by multiscale hybrid atom-level/coarse-grain simulations. J Phys Chem B 112:657-660

Neri M, Anselmi C, Cascella M, Maritan A, Carloni P (2005) Coarsegrained model of proteins incorporating atomistic detail of the active site. Phys Rev Lett 95:218102

Okvist M, Dey R, Sasso S, Grahn E, Kast P, Krengel U (2006) 1.6 Angstrom crystal structure of the secreted chorismate mutase from Mycobacterium tuberculosis: novel fold topology revealed. J Mol Biol 357:1483-1499

Orsi M, Sanderson WE, Essex JW (2009) Permeability of small molecules through a lipid bilayer: A multiscale simulation study. J Phys Chem B 113:12019-12029

Riniker S, van Gunsteren WF (2011) A simple, efficient polarizable coarse-grained water model for molecular dynamics simulation. J Chem Phys 134:084110

Riniker S, van Gunsteren WF (2012) Mixing coarse-grained and finegrained water in molecular dynamics simulations of a single system. J Chem Phys (in press)

Ryckaert J-P, Ciccotti G, Berendsen HJC (1977) Numerical-integration of Cartesian equations of motion of a system with contraints: molecular dynamics of n-alkanes. J Comput Phys 23:327-341

Rzepiela AJ, Louhivuori M, Peter C, Marrink SJ (2011) Hybrid simulations: combining atomistic and coarse-grained force fields using virtual sites. Phys Chem Chem Phys 13:10437-10448

Schmid N, Eichenberger AP, Choutko A, Riniker S, Winger M, Mark AE, van Gunsteren WF (2011) Definition and testing of the GROMOS force-field versions: 54A7 and 54B7. Eur Biophys J 40:843-856

Schmid N, Christ CD, Christen M, Eichenberger AP, van Gunsteren WF (2012) Architecture, implementation and parallelization of the GROMOS software for biomolecular simulation. Comput Phys Comm 183:890-903

Schindelin H, Jiang W, Inouye M, Heinemann U (1994) Crystal structure of CspA, the major cold shock protein of Escherichia coli. Proc Natl Acad Sci USA 91:5119-5123

Schwalbe H, Grimshaw S, Spencer A, Buch M, Boyd J, Dobson C, Redfield C, Smith L (2001) A refined solution structure of hen lysozyme determined using residual dipolar coupling data. Protein Sci 10:677-688

Shi Q, Izvekov S, Voth GA (2006) Mixed atomistic and coarsegrained molecular dynamics: simulation of membrane-bound ion channel. J Phys Chem B 110:15045-15048

Smith L, Sutcliffe M, Redfield C, Dobson C (1991) Analysis of F and $\chi_{1}$ torsion angles for hen lysozyme in solution from ${ }^{1} \mathrm{H}$ NMR spin-spin coupling constants. Biochemistry 30:986-996

Steiner D, Allison JR, van Gunsteren WF (2012) On the calculation of ${ }^{3} J_{\alpha \beta}$-coupling constants for side chains in proteins. J Biomol NMR. doi:10.1007/s10858-012-9634-5

Tironi IG, Sperb R, Smith PE, van Gunsteren WF (1995) A generalized reaction field method for molecular dynamics simulations. J Chem Phys 102:5451-5459

van Gunsteren WF, Billeter SR, Eising AA, Hünenberger PH, Krüger P, Mark AE, Scott WRP, Tironi IG (1996) Biomolecular simulation: the GROMOS96 manual and user guide; vdf Hochschulverlag AG an der ETH Zürich: Zürich, Groningen

Wüthrich K, Billeter M, Braun W (1983) Pseudo-structures for the 20 common amino acids for use in studies of protein conformations by measurements of intramolecular proton-proton distance constraints with nuclear magnetic resonance. J Mol Biol 169:949-961 